\title{
EL MODELO DE LOS CINCO GRANDES COMO PREDICTOR DE LA CONDUCTA AGRESIVA EN POBLACIÓN INFANTO-JUVENIL
}

\author{
Miguel Ángel CARrasco y María Victoria del Barrio \\ Facultad de Psicología, Universidad Nacional de Educación a Distancia
}

\begin{abstract}
Resumen: El presente trabajo estudia la relación entre el modelo de personalidad de los Cinco Grandes y la agresión física y verbal en un grupo de 686 sujetos (426 chicos y 260 chicas) de edades comprendidas entre 8 y 15 años. Los resultados muestran que las dimensiones de conciencia (especialmente en mujeres) y neuroticismo (especialmente en varones) son las más relevantes, aunque las dimensiones de amabilidad y extraversión también resultaron significativas principalmente en la agresión verbal. Se discute la necesidad de considerar diferentes tipos de agresión y la conveniencia de estudiar estas relaciones en chicos y chicas separadamente.
\end{abstract}

Palabras clave: Agresión, Cinco Grandes, personalidad, niños.

Five factor model of personality as predictor of aggressive behavior in children and adolescents

\begin{abstract}
The current paper studies the relationship between the Five Factor Model of personality and physical and verbal aggression in a sample of 686 subjects ( 426 boys and 260 girls) in ages between 8 and 15. The results show that conscientiousness (especially in girls) and neuroticism (especially in boys) are the most relevant, although agreeableness and extraversion were also significant dimensions, especially in verbal aggression. We discuss the need for considering different types of aggression and the convenience to study these relationships for boys and girls separately.
\end{abstract}

Key words: Aggression, Big Five, personality, children

\section{INTRODUCCIÓN}

La agresión infantil y juvenil se ha convertido en un problema prioritario cuyo incremento se ha constatado en diferentes estudios procedentes de diversos países (Achenbach, McConaughy y Howell, 1997; Olweus, 2003; Oñate y Piñuel, 2006; Tremblay, 2003). Los factores de riesgo de la conducta agresiva son muy numerosos y diversos, pero pueden agruparse en dos factores: sociales y personales (del Barrio, 2006). Los primeros tienen una solución principalmente política y los segundos, una intervención eminentemente psicológica. Si logramos aislar los factores personales que predicen precozmente la conducta agresiva,

Recibido 18 diciembre 2006; aceptado 25 enero 2007.

Correspondencia: Miguel Ángel Carrasco, Dpto. Personalidad Evaluación y Tratamientos Psicológicos, Facultad de Psicología, Juan del Rosal 10, 28040 Madrid. correo-e: macarrasco@psi.uned.es podremos actuar preventivamente. Existen dos modelos básicos que han delimitado la estructura de personalidad: el postulado por Eysenck que propone tres factores: psicoticismo, extraversión y neuroticismo (PEN) y el modelo de los cinco grandes (Big-Five, BF) que propone las dimensiones de amabilidad, apertura, neuroticismo, extraversión y conciencia (McCrae y Costa, 1985).

La relación entre estructura de personalidad y agresión, desde el modelo de Eysenck, aparece perfectamente clara: el factor que más fuertemente correlaciona con agresión es el psicoticismo y en segundo lugar el neuroticismo (Myrnard y Joseph, 1997; Slle y Rigby, 1993). En cuanto al factor extraversión aparece positiva y significativamente correlacionada con la agresión, aunque más débilmente que los anteriormente mencionados (Farell, 1992; Fonseca et al., 1995; Heaven 1993; Kerr, Au, Lindner, 2004). Estos son datos 
coincidentes con los hallados repetidamente en niños y adolescentes españoles (del Barrio, Moreno y López, 1997; del Barrio, Moreno y López, 2001). La menor potencia de la extraversión es interpretada por sus componentes tanto favorecedores (asertividad, actividad y exteriorización) como inhibidores (comunicación y amistad) de la agresión (Hampson y Goldberg, 2006). Es por esto que los datos que relacionan extraversión y agresividad no son monolíticos.

Cuando se parte del modelo de los Cinco Grandes los hallazgos son parecidos, pero más complejos. El neuroticismo, único factor negativo de este modelo, continúa comportándose de la misma manera que en el modelo de Eysenck para quien tenía un efecto multiplicador de los impulsos. Caprara y Pastorelli (1993), de acuerdo con Eysenck, sostienen que el neuroticismo propicia la falta de control y la impulsividad y en ello se basa la potente correlación encontrada con la conducta agresiva en población adolescente italiana. Esta misma relación se ha encontrado en niños holandeses cuando informan maestros aunque no aparece cuando los informantes son las madres (Kroes, Veerman y de Bruyn, 2005). El núcleo del problema, que conviene analizar despacio, está en la proliferación de los factores positivos de este modelo y en las fronteras difusas entre apertura, amabilidad y extraversión.

El factor amabilidad es el que se comporta más clara y sólidamente, puesto que presenta una correlación negativa con agresión y todas sus formas en todos los autores revisados. Así correlaciona con delincuencia (Heaven, 1996; Jonh, 1994), conducta antisocial (Loeber, Stouthamer-Loeber, Van Kammern y Farrington, 1989), agresión (Barbaranelli et al.1998; Graziano, Jensen-Campbell y Hair, 1996), problemas de conducta (Graciano y Ward, 1992; Victor, 1994), conflictividad (Graciano et al., 1996, 1997) y conducta antisocial (Shiner, 2000).

En el caso de la extraversión, como ocurría en el modelo de los Tres Factores, los datos ya no son tan uniformes. Hay autores que encuentran una relación positiva entre extraversión y agresión (Farrel, 1992; Kirkcaldy y Mooshage, 1993; Victor, 1994), sin embargo, en otros estu- dios como el realizado con adolescentes italianos, la relación entre extraversión y agresión es variable y, en todo caso, se suele dar con la agresión verbal, pero no con la física (Caprara, Barbaranelli y Zimbardo, 1996). Este mismo patrón se encuentra también en población juvenil española (Lemos, Fidalgo, Calvo y Menéndez, 1992).

Respecto del factor apertura pocos investigadores encuentran asociación con la agresión y, los que la encuentran, la hallan asociada con el rendimiento escolar (Loeber et al., 1989), lo que hace pensar que entre agresión y apertura juegue un papel mediador esencial esta variable, como apuntan también los resultados de Jonh, (1994), Victor, (1994), Barbaranelli et al. (1998) y Shiner (2000).

En el caso de la conciencia encontramos un panorama parecido al de apertura, pero el elemento mediador relevante es el acatamiento de normas y el control de la impulsividad (Loeber et al., 1989). Por ello, se encuentra una correlación negativa entre conciencia y conducta antisocial, pero no con otras formas de agresión (Heaven, 1996; Jonh, 1994).

Por otra parte, se advierte que los distintos factores de personalidad se asocian entre si de manera no homogénea. Lo más habitual es que amabilidad y conciencia actúen asociados: así los jóvenes violentos tienen niveles más bajos de conciencia y amabilidad (John, Caspi, Robins, Moffitt y Stouthamer-Loeber, 1994; Miller, Lynam y Leukefeld, 2003; Shiner, 2000; Tur et al., 2004). Pero también se han hallado asociaciones entre: neuroticismo y baja amabilidad (Steiner, Cauffman y Duxbury, 1999); extraversión con neuroticismo (Martín et al., 1999; Yamagata et al., 2006) o de estas dimensiones con baja conciencia (Hart, Hofmann, Edelstein y Keller, 1997) o baja amabilidad (Caprara et al., 1996). Incluso se ha encontrado un más amplio espectro de asociaciones que enlazan alto neuroticismo, alta extraversión, alto psicoticismo y alta sinceridad (Lemos et al., 1992).

Un estudio de meta-análisis reciente encuentra que la asociación entre baja amabilidad y alto neuroticismo es la combinación más habitualmente asociada a los problemas exteriorizados (Saulsman y Page, 2004). 
En un reciente e interesante trabajo (Lynam, Caspi, Moffit, Raine, Loeber, Stouthamer-Loeber, 2005) sobre adolescentes se comparan dos tipos de conducta agresiva en diferentes edades: «tendencia a la manipulación del otro» (Factor I) y «problemas de conducta y agresión» (Factor II). Sus resultados muestran que la amabilidad tiene un papel fundamental en la explicación de los dos tipos de problemas en todas las edades y la conciencia, especialmente en el segundo factor entre los adolescentes mayores.

El propósito de este trabajo es comprobar, en una muestra de niños y adolescentes españoles, cómo se comporta el modelo de los cinco grandes en relación con la conducta agresiva. Dado el carácter diferencial que la personalidad ha mostrado en relación con diferentes tipos de conducta agresiva y la presencia mayoritaria de esta conducta entre los varones, el objetivo se centra en analizar el valor predictivo de los cinco grandes tanto en la agresividad física como en la verbal en los grupos de chicos y chicas por separado.

\section{MÉTODO}

\section{Participantes}

La muestra estuvo constituida por 686 sujetos escolarizados (62\% varones y $38 \%$ mujeres) de edades comprendidas entre 8 y 15 años (media 11,82 y desviación tipo 2,21). Los sujetos fueron seleccionados mediante muestreo aleatorio simple de diferentes colegios del territorio nacional español. La participación en el estudio fue voluntaria y se requirió la previa autorización de los padres.

\section{Instrumentos}

Cuestionario de los Cinco Grandes para niños (BFQ-N; Barbaranelli, Caprara y Rabasca, 1998). Adaptación al castellano por Del Barrio, Carrasco y Holgado, 2006). El Cuestionario BFQ-N consta de 65 ítems acompañados de un escala tipo likert graduada en cinco niveles: 5 (casi siempre) a 1 (casi nunca). El instru- mento proporciona cinco escalas correspondientes a los factores de los cinco grandes: conciencia (20 ítems. Alfa de Cronbach $=0,88$; test-retest $=0,84$; p.ej., «Respeto las reglas y el orden»); apertura ( 8 ítems. Alfa de Cronbach $=0,84$; test-retest $=0,82$; p.ej., «Cuando el maestro explica algo, lo entiendo enseguida»); extraversión (10 ítems. Alfa de Cronbach = 0,79; test-retest $=0,71$; p.ej., «Me gusta hablar con los otros»); agradabilidad (10 ítems. Alfa de Cronbach $=0,80$; test-retest $=0,62$; p.ej., «Me comporto con los demás con mucha amabilidad») y neuroticismo (11 ítems. Alfa de Cronbach $=0,78$; test-retest $=0,77$; p.ej., $\ll \mathrm{Me}$ enfado con facilidad»).

Escala de Agresividad Física y Verbal (AFV, Caprara y Pastorelli, 1993). Adaptación española por Del Barrio, López-Martínez, MorenoRosset (2001). La escala de agresividad está compuesta de 20 elementos referidos a diferentes conductas de agresividad física ( 8 ítems. Alfa de Cronbach $=0,73$; p.ej., «Pego patadas y puñetazos»; «Doy empujones y pongo zancadillas») y agresividad verbal ( 8 ítems. Alfa de Cronbach $=0,67$; p.ej., «Amenazo a los otros»). Cada ítem está graduado en tres niveles según la frecuencia de emisión de la conducta a la que alude: 3 (a menudo); 2 (algunas veces); 1 (nunca). En el presente trabajo se utilizaron dos puntuaciones cada una de ellas procedentes respectivamente de la escala verbal y la escala física.

\section{Procedimiento}

Una vez que los sujetos eran autorizados a participar en el estudio fueron evaluados en pequeños grupos coincidentes con las clases de primaria y secundaria de los colegios estudiados. Antes de iniciar cada prueba se leían las instrucciones y se realizaba un ejemplo tipo. Durante la recogida de datos los sujetos eran atendidos por dos psicólogos quienes resolvían cualquier duda o problema que surgiera. Se les pedía que cumplimentaran las pruebas en silencio y que levantaran la mano si necesitaban alguna aclaración. Una vez finalizada la evaluación se procedió al análisis de los datos mediante el paquete estadístico SPSS 12.0. 


\section{RESULTADOS}

\section{Análisis de correlaciones}

En la Tabla 1 se presentan las correlaciones entre todas las medidas obtenidas. En relación con las correlaciones entre factores de personalidad y medidas de agresión, la agresividad tanto física como verbal, correlacionaron significativamente con todos los factores de personalidad, excepto la agresividad verbal con el factor extraversión. Como puede observarse en la mencionada tabla, destacan especialmente las correlaciones entre agresividad y conciencia (en sentido negativo) junto con las correlaciones entre agresividad y neuroticismo (en sentido positivo). En cuanto a las correlaciones entre factores de personalidad, éstas resultaron, en general, elevadas, principalmente entre los factores conciencia-apertura y conciencia-amabilidad. El factor que mostró mayor independencia respecto de lo otros fue el de neuroticismo. Por último, la edad correlacionó significativamente con las puntuaciones de agresividad y escasamente con los factores de personalidad.

\section{Diferencias por sexo}

Se estudiaron las diferencias por sexo, tanto en las medidas de agresividad como en las de personalidad. La comparación de medias en agresión, halladas mediante la $t$ de Student, indicó que los varones mostraron mayores niveles de agresividad tanto física $(t=3,91 ; p=$
$0,0001)$ como verbal $(t=4,04 ; p=0,0001)$. En cuanto a las diferencias por sexo en personali$\mathrm{dad}$, los varones presentaron significativamente mayores niveles de apertura $(t=2,82$; $p=0,005)$ y menores niveles de amabilidad $(t=-3,37 ; p=0,001)$. El resto de dimensiones resultaron equiparables entre chicos y chicas. En la tabla 2 aparecen los descriptivos (medias y desviaciones tipo) de las diferentes medidas junto con el estadístico correspondiente y su significación.

\section{Predicción de la conducta agresiva fisica y verbal por los Cinco Grandes}

Con el fin de hallar el valor predictivo del modelo de los Cinco Grandes se realizaron varios análisis de regresión múltiple. El método de introducción de las variables fue el método por pasos. Dada la elevada correlación existente entre los predictores y la consecuente multicolinealidad que esto supone, se centraron todas las variables mediante su estandarización.

Los dos primeros análisis de regresión (Tabla 3) se realizaron sobre el total de la muestra: primero, tomando la agresividad física como variable dependiente y segundo, la agresividad verbal. Todas las variables fueron incluidas conjuntamente en el análisis (variables de personalidad, edad y sexo). Los datos presentados se corresponden al último paso del análisis donde se muestran las variables significativas incluidas mediante el método mencionado (método por pasos). Los resultados mostraron que los porcentajes totales de varianza explicada por los facto-

Tabla 1. Correlaciones entre las variables

\begin{tabular}{|c|c|c|c|c|c|c|c|c|}
\hline & 1 & 2 & 3 & 4 & 5 & 6 & 7 & 8 \\
\hline 1. Conciencia & & & & & & & & \\
\hline 2. Apertura & $0,81 * *$ & & & & & & & \\
\hline 3. Extraversión & $0,56 * *$ & $0,40 * *$ & & & & & & \\
\hline 4. Amabilidad & $0,64 * *$ & $0,50 *$ & $0,57 * *$ & & & & & \\
\hline 5. Neuroticismo & $-0,31 * *$ & $-0,27 * *$ & $-0,25^{* *}$ & $-0,21 * *$ & & & & \\
\hline 6. Edad & $-0,25^{* *}$ & $-0,28$ & $0,09 *$ & 0,05 & $-0,02$ & & & \\
\hline 7. Agresividad física & $-0,40 * *$ & $-0,31 * *$ & $-0,10 * *$ & $-0,28 * *$ & $0,36 * *$ & 0,05 & & \\
\hline 8. Agresividad verbal & $-0,36 * *$ & $-0,27 * *$ & $-0,00$ & $-0,23 * *$ & $0,32 * *$ & $0,19 * *$ & $0,71 *$ & \\
\hline 9. Agresividad total & $-0,40 * *$ & $-0,31 * *$ & $-0,04$ & $-0,27 * *$ & $0,35 * *$ & $0,15^{* *}$ & $0,90 *$ & $0,93 * *$ \\
\hline
\end{tabular}

Nota. $* p<0,05 ; * * p<0,01$. 
Tabla 2. Diferencias por sexo en las diferentes medidas de personalidad y agresión

\begin{tabular}{|c|c|c|c|c|c|}
\hline & Sexo & $\mathrm{N}$ & Media & $D T$ & $t$ \\
\hline \multirow[t]{2}{*}{ Conciencia } & 1 & 426 & 70,44 & 13,53 & \\
\hline & 2 & 260 & 71,50 & 13,28 & $-0,99$ \\
\hline \multirow[t]{2}{*}{ Apertura } & 1 & 426 & 27,58 & 6,34 & \\
\hline & 2 & 260 & 26,17 & 6,33 & $2,82 * *$ \\
\hline \multirow[t]{2}{*}{ Extraversión } & 1 & 426 & 40,43 & 6,15 & \\
\hline & 2 & 260 & 40,93 & 5,93 & $-1,04$ \\
\hline \multirow[t]{2}{*}{ Amabilidad } & 1 & 426 & 36,12 & 6,57 & \\
\hline & 2 & 260 & 37,83 & 6,20 & $-3,37 * *$ \\
\hline \multirow[t]{2}{*}{ Neuroticismo } & 1 & 426 & 27,23 & 7,39 & \\
\hline & 2 & 260 & 27,77 & 7,67 & $-0,91$ \\
\hline \multirow[t]{2}{*}{ Agresividad Física } & 1 & 426 & 11,15 & 2,62 & \\
\hline & 2 & 260 & 10,37 & 2,33 & $3,91 * *$ \\
\hline \multirow[t]{2}{*}{ Agresividad Verbal } & 1 & 426 & 14,15 & 2,95 & \\
\hline & 2 & 260 & 13,26 & 2,55 & $4,04 * *$ \\
\hline
\end{tabular}

Nota. Categorías de la variable sexo: 1 niño; 2 niña. ${ }^{*} p<0,05 ; * * p<0,01$.

res de personalidad fueron respectivamente: el $26,2 \%$ para la agresividad física y el 26,9\% para la agresividad verbal. En ambos casos, los factores que obtuvieron mayor valor predictivo fueron conciencia y neuroticismo y, en menor medida, extraversión y amabilidad. Estos últimos (especialmente la extraversión) se mostraron superiores en la predicción de la agresividad verbal que de la física. La apertura quedó excluida del análisis, posiblemente por su elevada correlación con el factor conciencia. De acuerdo con el signo de los coeficientes estandarizados correspondientes, los niveles elevados de neuroticismo o extraversión favorecen la conducta agresiva (física y verbal) mientras que la elevada conciencia o amabilidad la inhiben. No obstante, la baja conciencia y el elevado neuroticismo predicen más potentemente la conducta agresiva física y, en cambio, la elevada extraversión y baja amabilidad la conducta agresiva verbal.

El sexo predijo la agresividad tanto física como verbal aproximadamente con un $2 \%$ de la varianza explicada. La edad sólo resultó significativa en la predicción de la agresividad verbal con un porcentaje del $0,6 \%$.

Dado que los niveles de agresión (principalmente la agresión física) se muestran sistemá-

Tabla 3. Análisis de regresión múltiple (paso a paso) de los Cinco Grandes sobre las variables de agresividad en la muestra total

\begin{tabular}{|c|c|c|c|c|c|c|}
\hline & \multicolumn{3}{|c|}{ Agresión física } & \multicolumn{3}{|c|}{ Agresión verbal } \\
\hline & $B$ & $t$ & $R_{\text {ajust. }}^{2}$ & $B$ & $t$ & $R_{\text {ajust. }}^{2}$ \\
\hline Conciencia & $-0,353$ & $-7,45^{* *}$ & 0,154 & $-0,317$ & $-6,07 * *$ & 0,125 \\
\hline Apertura & - & - & - & - & - & - \\
\hline Extraversión & 0,233 & $5,36^{* *}$ & 0,024 & 0,319 & $7,21 * *$ & 0,052 \\
\hline Amabilidad & $-0,107$ & $-2,27^{*}$ & 0,005 & $-0,14$ & $-2,95 * *$ & 0,009 \\
\hline Neuroticismo & 0,284 & $7,89 * *$ & 0,058 & 0,272 & $7,55 * *$ & 0,055 \\
\hline Edad & - & - & - & 0,116 & $3,11 * *$ & 0,006 \\
\hline Sexo & $-0,138$ & $-4,05 * *$ & 0,02 & $-0,14$ & $-4,26 * *$ & 0,022 \\
\hline
\end{tabular}

Nota. Todos los predictores fueron centrados. Datos correspondientes al último paso de inclusión. $B=$ Coeficientes estandarizados. $* p<0,05 ; * * p<0,01$. 
ticamente superiores en los varones respecto de las mujeres, se realizaron análisis de regresión para cada uno de los grupos por separado. Se trataba de averiguar si se mantienen los mismos predictores en ambos grupos o, en cambio, éstos predicen de manera diferencial la agresividad física y verbal de chicos y chicas (tabla 4). Como en el análisis anterior, los datos presentados se corresponden al último paso del análisis donde se muestran las variables significativas incluidas mediante el método por pasos. Los resultados aparecidos muestran que tanto los predictores como los valores de los mismos difieren entre un grupo y otro: a) mientras que el neuroticismo es el mejor predictor en el grupo de los varones, tanto para la agresión física como para la verbal, en el grupo de las mujeres, el mejor predictor es la conciencia; b) la amabilidad, funciona como un predictor de la agresividad (especialmente la física) en el caso de los chicos, pero no de las chicas; c) la conciencia pierde valor predictivo en la agresividad física a favor del neuroticismo (no en la agresividad verbal) sólo en el grupo de los chicos, pero no en el de las chicas.

Considerando globalmente el valor predictivo de los factores de personalidad, éstos explicaban porcentajes de varianza explicada entre el 23 y el $30 \%$ : en los varones, $24,7 \%$ para la agresión física y $23,3 \%$ para la agresión verbal; en las mujeres, $28 \%$ para la agresión física y $30 \%$ para la agresión verbal.

\section{DISCUSIÓN}

La estructura de personalidad de los cinco grandes, tal y como aparece en numerosos trabajos (Barbaranelli et al., 1998; Caprara et al, 1993; Miller et al., 2003; Lynam, 2005), predijo significativamente la conducta agresiva tanto física como verbal. En el presente estudio, los factores de personalidad que obtuvieron mayor potencia en ambos tipos de agresividad fueron los de conciencia y neuroticismo, especialmente el primero, y en menor medida los de extraversión y amabilidad. La apertura no contribuyó a explicar ninguno de los tipos de agresividad estudiados.

La conciencia aparece frecuentemente asociada con bajos niveles de agresividad (Loeber et al., 1989; John et al., 1994; Shiner, 2000; Tur et al., 2004) y ello está perfectamente justificado dado sus componentes de reflexividad, responsabilidad y control como inhibidores de las conductas exteriorizadas. De acuerdo con resultados procedentes de otros estudios (Caprara et al, 1993; Kroes et al., 2005; Martín et al., 1999) el neuroticismo, cuyas características incluyen inestabilidad, impulsividad e ira, en definitiva, emocionalidad, es un potenciador de la respuesta agresiva. Estos resultados son congruentes con los hallados en el presente estudio, si bien en este caso y referido al total de la muestra, el peso del neuroticismo obtiene una potencia menor que el factor conciencia.

A pesar de que la amabilidad ha ocupado un papel predominante en la explicación de la

Tabla 4. Análisis de regresión múltiple (paso a paso) de los Cinco Grandes sobre las variables de agresividad en cada uno de los sexos

\begin{tabular}{|c|c|c|c|c|c|c|c|c|c|c|c|c|}
\hline & \multicolumn{6}{|c|}{ Agresión física } & \multicolumn{6}{|c|}{ Agresión verbal } \\
\hline & \multicolumn{3}{|c|}{ Varones $(n=426)$} & \multicolumn{3}{|c|}{ Mujeres $(n=260)$} & \multicolumn{3}{|c|}{ Varones $(n=426)$} & \multicolumn{3}{|c|}{ Mujeres $(n=260)$} \\
\hline & $B$ & $t$ & $R_{\text {ajust. }}^{2}$ & $B$ & $t$ & $R_{\text {ajust. }}^{2}$ & $B$ & $t$ & $R_{\text {ajust. }}^{2}$ & $B$ & $t$ & $R_{\text {ajust. }}^{2}$ \\
\hline Conciencia & $-0,228$ & $-3,57 * *$ & 0,022 & $-5,21$ & $-8,03 *$ & 0,199 & $-0,211$ & $-3,00 * *$ & 0,037 & $-0,57$ & $-8,94$ & 0,186 \\
\hline Apertura & - & - & - & - & - & - & - & - & - & - & - & - \\
\hline Extraversión & 0,013 & $3,77 * *$ & 0,013 & 0,270 & $4,24 * *$ & 0,037 & 0,289 & $5,02 * *$ & 0,174 & 0,375 & $5,97 * *$ & 0,082 \\
\hline Amabilidad & $-0,211$ & $-3,48 * *$ & 0,055 & - & - & - & $-0,189$ & $-3,04 * *$ & 0,012 & - & - & - \\
\hline Neuroticismo & 0,322 & $6,89 * *$ & 0,157 & 0,230 & $4,05^{* *}$ & 0,044 & 0,319 & $6,68 * *$ & 0,122 & 0,206 & $3,69 * *$ & 0,035 \\
\hline Edad & - & - & - & - & - & - & 0,122 & $2,52 * *$ & 0,010 & - & - & - \\
\hline
\end{tabular}

Nota. Todos los predictores fueron centrados. Datos correspondientes al último paso de inclusión; $B=$ Coeficientes estandarizados. ${ }^{*} p<0,05 ; * * p<0,01$. 
agresividad por su carácter interpersonal asociado a componentes de empatía, cooperación, franqueza, altruismo y actitud conciliadora (Graciano et al., 1996; Miller et al., 2003; Shiner, 2000), en los resultados hallados, su papel quedó desplazado por los factores de neuroticismo y conciencia. No obstante, se asoció significativamente con la agresión tal y como ocurrió con la extraversión. Respecto a este último factor, los presentes datos muestran su asociación significativa con la agresión, sin embargo, la vinculación entre extraversión y conductas antisociales ha arrojado resultados muy controvertidos que se han explicado, bien por el tipo de conducta antisocial (Carrasco, Barker, Tremblay y Vítaro, 2006), bien por la intensidad de esta dimensión o bien por su asociación con otro tipo de dimensiones de personalidad (Martín et al., 1999; Yamagata et al., 2006).

Todo lo expuesto anteriormente afecta a la agresividad considerada globalmente, es decir, a la agresividad física y verbal en su conjunto. Sin embargo, si se comparan las características de personalidad en cada uno de los tipos de agresividad por separado, extraversión y amabilidad obtienen mayor relevancia en la agresión verbal que en la física. Podría concluirse, que los sujetos con mayores niveles de neuroticismo y baja conciencia son más vulnerables al desarrollo de conductas agresivas, pero si además poseen altos niveles de extraversión y baja amabilidad, tenderán a manifestar mayores niveles de agresión verbal. Estos resultados concuerdan en lo que respecta a amabilidad con los resultados de Lynam et al. (2005), pero no con respecto a extraversión. Este dato apoya la conveniencia de tener en cuenta diferentes tipos de conducta agresiva en su relación con factores de personalidad (Carrasco et al, 2006; Lynam et al., 2005; Miller et al., 2001).

En los resultados que se aportan en este trabajo aparece un claro patrón diferencial entre sexos, según el cual los chicos muestran más agresión que las chicas. Por ello, es interesante resaltar las diferencias aparecidas entre ambos en relación con conducta agresiva y personalidad: mientras que el neuroticismo es la dimensión más explicativa entre los varones principalmente en la agresividad física, la conciencia lo es entre las mujeres en cualquiera de sus manifestaciones agresivas (física y verbal). Es coherente que en los varones pesen más aspectos temperamentales de la personalidad, como el neuroticismo, frente aspectos instrumentales como conciencia (Costa y McCrae, 1980; McCrae y Costa, 1991) dado el supuesto origen biológico de la agresividad física en varones (Carey y Goldman, 1997) así como del neuroticismo (Watson y Clark, 1992). Por otra parte, la amabilidad es una característica explicativa de la agresión (especialmente física) en los varones, pero no en las mujeres. Dado que la amabilidad es una característica más predominante entre éstas (Budaev, 1999; Carrasco, Holgado y del Barrio, 2006) y, por tanto, más homogénea entre este grupo de población, puede ser que no permita discernir su relevancia en relación con la agresión cuando se toma al grupo de chicas como un todo. No obstante, los escasos estudios que han explorado estas relaciones no permiten hacer una comparación más exhaustiva. Algunos trabajos recientes han hallado que las aportaciones del sexo en relación con la personalidad y la agresión es escaso o poco relevante (Miller et al., 2003), seguramente por la colinealidad de la variable sexo con las variables de personalidad.

La apertura, como se ha indicado, no resultó significativa en ninguno de los análisis realizados en este trabajo. Aunque la apertura en relación con la conducta agresiva no ha mostrado resultados consistentes, en nuestro caso, este resultado es claramente explicado por la elevada correlación que esta variable mantiene con la dimensión conciencia y cuyo denominador común está claramente asociado con los contenidos escolares (interés por las materias escolares en el caso de la apertura y cumplimiento y responsabilidad de las mismas en el caso de la conciencia). Si bien, la inconsistencia de la asociación entre apertura y agresividad deba ser explicada en relación con la operativización y delimitación de este factor respecto del resto de dimensiones de personalidad. Como han recogido otros trabajos, la apertura a pesar de ser conceptualmente autónoma, es una dimensión que se solapa con extraversión (Aluja, García y García, 2002) y Conciencia (Scholté y De Bruyn, 2004).

De lo anteriormente expuesto, se puede concluir que hay dos estilos favorecedores de la 
conducta agresiva y que agrupan distintos factores, similar al propuesto en el metaanálisis de Miller y Lynam (2001): uno vinculado al neuroticismo, de carácter emocional y otro, a baja conciencia o ausencia de cognición o control.

Finalmente, una mención a algunas recomendaciones para futuros trabajos que permitan superar las limitaciones del presente estudio. Entre ellas cabe resaltar la conveniencia de: a) analizar las conductas agresivas evaluadas por otros informantes para determinar las diferencias de apreciación que influyen en los datos; b) analizar longitudinalmente los datos para obtener las trayectorias de estas relaciones y c) la ampliación del rango de edad con el fin de analizar en cada subgrupo las diferencias entre sexo y determinar más claramente el efecto de la edad en las relaciones estudiadas.

\section{REFERENCIAS}

Achenbach, T.M., McConaughy, S.H., y Howell, C.T. (1997). Child/adolescent behavioral and emotional problems: implications for across-informant correlations for situational specificity. Psychological Bulleting, 101, 213-132.

Aluja, A., García, O., y García, L. (2002). A comparative study of Zuckerman's three structural models for personality through the NEO-PI-R, ZKPQ-III-R, EPQ-RS and Goldberg's 50 bipolar adjectives. Personality and Individual Differences, 33, 713-725.

Barbaranelli, C., Caprara, G.V., y Rabasca, A. (1998). Manuale del BFQ-C. Big Five Questionnaire Children. O.S. Organizzaaioni Speciali-Firenze.

Budaev, S.V. (1999). Sex differences in the big five personality factors: testing an evolutionary hypothesis. Personality and Individual Differences, 26, 801-813.

Caprara, G.V., Barbaranelli, C., y Zimbardo, P. (1996). Understanding the complexity of human aggression: affective, cognitive, and social dimensions of individual differences in propensity toward aggression. European Journal of Personality, 10, 133-155.

Caprara, G.V., y Pastorelli, C. (1993). Early emotional inestability, prosocial behaviour and aggression: some methodological aspects. European Journal of Personality, 7, 19-36.

Carey, G., y Goldman, D. (1997). The genetics of antisocial behavior. En D. Staff, J. Breiling, J. Maser (Eds.), Advances in clinical child psychology (pp. 137-175). New York: Plenum.
Carrasco, M.V., Barker, E.D., Tremblay, R.E., y Vitaro, F. (2006). Eysenk's personality as predictors of male adolescent trajectories of physical agresión, theft and vandalism. Personality and Individual Differences, 41, 1309-1320.

Costa, P.T., y McCrae, R.R. (1980). Influence of extraversion and neuroticism on subjective well-being: happy and unhappy people. Journal of Personality and Social Psychology, 38, 668-678.

Chorpita, B.F., Plummer, C.M., y Moffitt, C.E. (2000): Relations of tripartite dimensions of emotion to childhood anxiety and mood disorders. Journal of Abnormal Child Psychology, 28, 299-310.

Davis, H., y Carr, M. (2001). Gender differences in mathematics strategy use: The influence of temperament. Learning and Individual Differences, 13, 83-95.

Del Barrio, V. (2002). Emociones Infantiles. Madrid: Pirámide.

Del Barrio, V. (2006). Prólogo. En V. del Barrio, M.A. Carrasco y F.P. Holgado, BFQ-NA Cuestionario Big Five de personalidad para niños y adolescentes. Madrid: TEA.

Del Barrio, V., Carrasco, M.A., y Holgado, F.P (2006). BFQ-NA Cuestionario Big Five de personalidad para niños y adolescentes. Madrid: TEA.

Del Barrio, V., Carrasco, M.A., y Holgado, F.P. (2006). Análisis transversal de los Cinco Factores de personalidad en distintos grupos de sexo y edad de una muestra de niños españoles. Revista Latinoamericana, 38 , 567-577.

Del Barrio, V., Moreno, C., y López, R. (1997). Anxiety, depresión and personality structure. Personality and Individual Differences, 23, 327-335.

Del Barrio, V., Moreno, C., y López, R. (2001). Evaluación de la agresión e inestabilidad emocional en niños españoles y su relación con la depresión. Clínica y Salud, 12, 33-50.

Farrell, M. (1992). Personality and anti-social behaviour among emotionally behaviourally disturbed boys. Personality and Individual Differences, 13, 511-517.

Fonseca, A., y Yule, W. (1995). Personality and antisocial behavior in children and adolescents: an enquiry into Eysenck's and Gray's theories. Journal of Abnormal Child Psychology, 23, 767-781.

Graziano, W., Jensen-Campbell, L., y Finch, J. (1997). The self as a mediator between personality and adjustment. Journal of Personality and Social Psychology, 2, 392-404.

Graziano, W., Jensen-Campbell, L., y Hair, E. (1996). Perceiving interpersonal conflict and reacting to it: the case for agreeableness. Journal of Personality and Social Psychology, 70, 820-835.

Graziano, W., y Ward, D. (1992). Probing the big five in adolescence: personality and adjustment during a developmental transition. Journal of Personality, 60, 425-439. 
Hampson, S.E., y Goldberg, LR. (2006). A first large cohirt study of personality trait stability over the 40 years between elementary school and midlife. Journal of Personality and Social Psychology, 91, 763-779.

Hart., D., Hofmann, V., Edelstein, W., y Keller, M. (1997). The relation of childhood personality types to adolescent behavior and development: a longitudinal study of icelandic Children. Developmental Psychology, 33, 195-205.

Heaven , P. (1993). Personality predictors of self-reported delinquency. Personality and Individual Differences, 14, 67-76.

Heaven, P. (1996). Personality and self-reported delinquency: analysis of the «Big Five» personality dimensions. Personality and Individual Differences, 20, 47-54.

Jang, KL., Livesley, WJ., Ando, J. Yamagata, S. et al. (2006). Behavioral genetics of the higher-order factor of Big Five. Personality and individual Differences, 41, 261-272.

John, O., Caspi, A., Robins, R., Moffitt, T., y StouthamerLoeber, M. (1994). The «Little five»: Exploring the nomological network of the Five-Factor Model of Personality in adolescent boys. Child Development, 65, 160-178.

Kerr, J.H., Au, C.K.F. y Lindner, K.J. (2004). Motivattion and level of risk in male and female recreational sport participation. Personality and Individual Differences, 37, 1245-1253.

Kirkcaldy, B.D., y Mooshage, B. (1993). Personality profiles of conduct and emotionally disordered adolescents. Personality and Individual Differences, 15, 95-96.

Kroes, G., Veerman, JW., y de Bruyn, E. (2005). The impact of big five personality traits on reports of child behavior problems by different informants. Journal of Abnormal Child Psychology, 33, 231-240.

Lemos, S., Fidalgo, A., Calvo, P., y Menéndez, P. (1992). Validación de la escala de psicopatología infanto-juvenil YSR. Clínica y Salud, 3, 183-194.

Loeber, R., Stouthamer-Loeber, M., Van Kammern, W., y Farrington, D. (1989). Development of a new measure for self-reported antisocial behavior for young children: Prevalence and reliability. En M.Klein (Ed.), Cross-national research in self-reported crime and delinquency (pp. 203-225). Norwell, MA: Kluwer Academic Press.

Lynam, D., Caspi, A., Moffit, T., Raine, A., Loeber, R., y Stouthamer-Loeber, M. (2005). Adolescent Psychopathology and big-five: Results from two samples. Journal of Abnormal Child Psychology, 33, 431-443

Madsen, L., Parson, S., y Grubin, D. (2006). Relationship between the five-factor model and DSM personality disorders in a sample of child molesters. Personality and individual Differences, 40, 227-236.
Martin, R., Wan, Ch., David, J., Wegner, E., Olson, B. y Watson, D. (1999). Style of anger expression: relation to expressivity, personality and health. Personality and Social Psychology Bulletin, 25, 1196-1207.

McCrae, R.R., y Costa, P.T. Jr. (1991). Adding Liebe and Arbeit: The full five-factor model and well-being. Personality and Social Psychology Bulletin, 17, 227-232.

McCrae, R.R., y Costa, P.T. (1985). Comparison of EPI and psychoticism scales with measures of the five-factor theory of personality. Personality and individual Differences, 6, 587-597.

Miller, J., Lynam, D., y Leukefeld, C. (2003). Examining antisocial behaviour through the lens of the Five Factor Model of Personality. Aggressive Behavior, 29, 497-514.

Mynard, H., y Joseph, S. (1997). Bully/victim problems and their association with Eysenck's personality dimensions in 8 to 13 year-olds. British Journal of Educational Psychology, 67, 51-54.

Olweus, D. (2005). A useful evaluation design and effects of the Olweus Bullying Prevention Program. Psychology, Crime and Law, 11, 389-402.

Oñate, A., y Piñuel, I. (2006). Estudio Cisneros X. Violencia y acoso escolar en España. Madrid: Instituto de Innovación Educativa y Desarrollo Directivo.

Saulsman, LM., y Page, AC. (2004). The five-factor model and personality disorder empirical literature: a metanalytic review. Clinical Psychology Review, 23, 1055-1085.

Scholte, R., y De Bruyn, E. (2004). Comparison of the Giant Three and the Big Five in early adolescents. Personality and Individual Differences, 36, 1353-1371.

Shiner, R.L. (2000). Linking childhood personality with adaptation: Evidence for continuity and change across time into late adolescence. Journal of Personality and Social Psychology, 28, 310-325.

Slle, P.T., y Rigby, K. (1993). The relationship of Eysenck's personality factors and self-esteem to bully/victim behaviour in Australian school boys. Personality and Individual Differences, 14, 371-373.

Sobral, J., Romero, E., Luengo, A., y Marzoa, J. (2000). Personalidad, conducta antisocial: amplificadores individuales de los efectos contextuales. Psicothema, 4 , 661-670.

Steiner, H., Cauffman, E., y Duxbury, E. (1999). Personality traits in juvenile delinquents: relation to criminal behavior and recidivism. Journal of the American Academy Child Adolescent Psychiatry, 38, 256-262.

Tremblay, R. (2003): Los orígenes de la violencia en los jóvenes, Acción Psicológica, 2, 63-72.

Tur, A., Mestre, V., y del Barrio, M.V. (2004). Factores moduladores de la conducta agresiva y prosocial, el efecto de los hábitos de crianza. Ansiedad y Estrés, 10, 75-88.

Victor, J. (1994). The five factor model applied to individual differences in school behavior. En C.F. Halverson, G.A. Kohnstamm y R. Martin (Eds.), The developing structu- 
re of temperament and personality from infancy to adulthood (pp. 355-366). Hillsdales, New Jersey: LEA.

Watson, D., y Clark, L.A. (1992). On traits and temperament: general and specific factors of emotional experience and their relation to the five factor model. Journal of Personality, 60, 441-446.

Watson, D., y Clark, L.A. (1997). Measurement and mismeasurement of mood: recurrent and emergent issues. Journal of Personality Assessment, 68, 267-296.
Williams, D.G. (1990). Effects of Psychoticism, Extraversion and Neuroticism in courrent mood: A statistical review of six studies. Personality and Individual Differences, 11, 615-630.

Yamagata, S., Suzuki, A., Ando, J. et al (2006). Is genetic structure of human personality universal. A crosscultural twin study from North America Europe and Asia. Journal of Personality and Social Psychology, 90, 987-998. 